\title{
Manual training of mental rotation
}

\author{
Gunnar Wiedenbauer, Juliane Schmid, and \\ Petra Jansen-Osmann \\ Heinrich-Heine-University of Duesseldorf, Germany
}

\begin{abstract}
In mental rotation tasks, reaction time increases linearly with increasing angular disparity (Shepard \& Metzler, 1971). Extensive repetition of mental rotation has been shown to reduce reaction times (e.g., Tarr \& Pinker, 1989). This training effect, however, seems to be based on the retrieval of memory representations rather than on a faster execution of the rotation process itself (e.g., Heil, Roesler, Link, \& Bajric, 1998). The main purpose of the present experiments was to investigate whether mental rotation can be trained by a manual rotation task in a virtual environment. Since manual rotation does visualise the process of mental rotation and as mental rotation is assumed to be a covert motor rotation (Wexler, Kosslyn, $\&$ Berthoz, 1998), it should train the rotation process itself and thus should not be memory based. In Experiment 1, a new virtual mental rotation test was validated successfully. Experiment 2 shows that manual training of mental rotation in a virtual environment is effective but limited to trained objects.
\end{abstract}

The concept of mental rotation was introduced by Shepard and Metzler in 1971. In their groundbreaking study, participants had to decide whether two three-dimensional block stimuli rotated in space were the same or were mirror-reflected. Their results showed that response time increased linearly with increasing angular disparity between the two objects. Shepard and Metzler concluded that participants solved the task by mentally rotating one of the objects. Numerous further studies employed the Shepard and Metzler paradigm using two- and three-dimensional stimuli (e.g., Cooper \& Shepard, 1973; Shepard \& Metzler, 1988) and covered developmental (e.g., Marmor, 1975), neurophysiological (e.g., Barnes et al., 2000), and differential issues (e.g., sex differences: Voyer, Voyer, \& Bryden, 1995).

Correspondence should be addressed to Gunnar Wiedenbauer, Institut für Experimentelle Psychologie, Heinrich-Heine-Universität, Universitätsstr. 1, 40225 Düsseldorf, Germany. E-mail: gunnar.wiedenbauer@uni-duesseldorf.de

This work was supported by grant JA 889/3-1 of the Deutsche Forschungsgemeinschaft (German Research Foundation) to Petra Jansen-Osmann. We would like to thank Cornelia Delbos, Jan Dombrowski, Martin Heil, Stefanie Holdmann, Kristina Küper, Léonie Lange, and Monika Renvert for their help. Furthermore, we thank Philippe Peigneux, Albert A. Rizzo, and an anonymous reviewer for helpful comments on an earlier draft of this paper.

(C) 2007 Psychology Press, an imprint of the Taylor and Francis Group, an informa business http://www.psypress.com/ecp

DOI: $10.1080 / 09541440600709906$ 
Many studies showed that mental rotation can be trained by extensive repetition of mental rotations: Reaction time decreases with training (e.g., Kail \& Park, 1990; Kaushall \& Parsons, 1981). However, there is evidence indicating that the mental rotation process itself is not improved by mental rotation training (Heil, Roesler, Link, \& Bajric, 1998; Tarr \& Pinker, 1989). The main goal of the study presented here was to investigate effects of a virtual manual rotation training on mental rotation.

In a mental rotation trainings study, Tarr and Pinker (1989) showed that the overall reaction time as well as the slope of the linear function between reaction time and angular disparity decreased with extensive training. Their results, however, also demonstrated that it cannot be inferred from the decrease of the slope that the process of mental rotation is executed faster after training since the effect of orientation nearly disappeared and training had no effect on practiced objects presented in new, unlearned orientations. The results of Tarr and Pinker indicated that mental rotation was replaced by the retrieval of stored memory representations. Similar results were found by Heil et al. (1998). Their participants executed three test sessions of mental rotation. Before the second session, the practice group participated in four training sessions of mental rotation. Reaction times decreased from one training session to the next. The practice group benefited from the training for learned objects, but only when these objects were presented in exactly the same orientation as during training. Furthermore, reaction times for new objects in learned orientations did not decrease. Thus, the effect of mental rotation training seems to be highly stimulus and perspective specific. The data therefore suggest that the mental rotation process is not executed more rapidly after training but replaced by memory retrieval.

Overall, practising mental rotation does have a training effect, even if this effect does not seem to be due to a faster execution of the mental rotation process. However, it cannot be inferred, that the mental rotation process itself is not trainable. Manual rotation might be the appropriate task to train mental rotation since mental rotation is assumed to be a covert motor rotation (Wexler, Kosslyn, \& Berthoz, 1998) and shares the same processes with manual rotation (Wohlschlaeger \& Wohlschlaeger, 1998). In the study of Wexler et al., participants had to rotate a joystick while executing a mental rotation task. The reaction times for mental rotation trials which were incompatible with the manual rotation (mental rotation clockwise while manual rotation counterclockwise and vice versa) were slower than for compatible rotations. Furthermore, in the compatible condition, a change in the speed of the manual rotation resulted in an analogous shift in the time taken to complete the mental rotation.

A close relationship between mental and manual rotation has also been shown in a similar study by Wohlschlaeger and Wohlschlaeger (1998). Participants had to match a block figure to one of two comparison figures. 
While one group of participants mentally rotated the stimuli, the other group rotated the stimuli manually by using a knob. Reaction time of mental as well as manual rotation increased with increasing angular disparity between the two stimuli and both mental and manual rotation were influenced by the axis of rotation in the same manner. In a second experiment, in which the influence of various hand movements on mental rotation was assessed, an effect of hand rotation was only found when the axis of rotation was the same for hand movement and mental rotation: The mental rotation speed was slowed down when the directions of the motor and mental rotation were incompatible. No influence was found when a translational hand movement was demanded while rotating the stimuli mentally.

Further evidence that mental and manual rotation share the same processes stems from neuroimaging studies in which participants had to mentally rotate body parts: Motor and premotor areas which are involved in the execution of movements are also activated during mental rotation (e.g., Kosslyn, DiGirolamo, Thompson, \& Alpert, 1998; Wraga, Thompson, Alpert, \& Kosslyn, 2003). These findings are supported by neurophysiological studies with monkeys (Hietanen \& Perrett, 1996) and humans (Barnes et al., 2000).

Thus, the close relation between mental and manual rotation and the fact that the process of the rotation is visualised in a manual task lead to the assumption that manual rotation training should improve the performance of mental rotation. Furthermore, manual training might improve the rotation process itself and therefore not rely on the retrieval of memory representations and consequently not be object specific.

A first attempt to improve mental rotation ability by manual training was made by Rizzo and colleagues (Larson et al., 1999; Parsons et al., 2004; Rizzo et al., 2001). In their manual training of spatial rotation in a virtual reality, participants had to rotate a block figure into the orientation of the target figure and superimpose it on the target figure. The stimuli were presented on a large-screen stereoscopic display and could be rotated both in depth and picture plane by manually rotating a sphere shaped "cyberprop". Mental rotation ability was assessed by using the Mental Rotation Test (MRT; Vandenberg \& Kuse, 1978), a paper and pencil test. The overall improvement in the MRT did not differ between the training group and a control group that received a nonspatial filler task. Only a post hoc analysis showed that poor mental rotators scored higher on the MRT following training. Ceiling effects might have prevented the finding of a training effect for good mental rotators. However, we suppose that the lack of an overall training effect is due to the various differences between training and test, such as the change from a three-dimensional virtual reality setup to a paper and pencil test with line drawings or the change from one to four comparison figures. Furthermore, the conclusions that can be drawn from 
the MRT are limited: Firstly, due to the time limit imposed, the test score does not differentiate between errors and response time. Secondly, the angular disparity between the standard figure and the comparison figures is not varied in a systematic way and thus does not allow for a computation of the mental rotation function.

In order to illustrate an effect of manual training on mental rotation ability, the mental rotation test used should be as similar to the training as possible. To investigate the basis of potential training effects, a mental rotation test which assesses the mental rotation performance in more detail - namely the assessment of reaction times and errors depending on the amount of angular disparity - and which differentiates between trained and untrained objects is necessary.

Therefore, a computer-based mental rotation test, which fulfils the requirements described above, and a manual rotation training were constructed. Before investigating the effect of the manual training on mental rotation ability (Experiment 2), the virtual mental rotation test was validated in Experiment 1. We exclusively used stimuli that were rotated in picture plane since Shepard and Metzler (1971) could show that both types of rotations (picture plane and depth rotations) produced the similar linear relationship between angular disparity and reaction times.

\section{EXPERIMENT 1}

The purpose of the first experiment was to validate our virtual mental rotation test (VMRT). Concurrent validity was tested by correlating the VMRT with the MRT (Vandenberg \& Kuse, 1978), which is a well recognized test instrument to measure mental rotation ability. Furthermore we expected to find the linearly increasing function between reaction time and angular disparity usually found in mental rotation.

\section{Method}

Participants. 107 subjects participated in the experiment, 55 of whom were female. Their age ranged from 17 to 36 years $(M=22.2)$. 83 participants were university students (46 of whom studied psychology) and the remaining 24 were pupils or employees. The vision of all subjects was corrected to 20/20. Five participants were dismissed due to an overall error rate of above $40 \%$ in the VMRT. This resulted in a total of 102 participants (half of whom were female).

Materials. The MRT (redrawn version of Peters, Laeng, Latham, \& Jackson, 1995; Vandenberg \& Kuse, 1978), a paper and pencil test of mental rotation ability, was used. In this test, the participants' task is to decide 
which two of four given line drawings of block figures match the standard figure. "Different" comparison figures are either mirror images of the standard figure or completely different block figures. "Same" and "different" figures are rotated in the picture plane and/or in depth. The MRT consists of two 12-item sections each with a 3 minute time limit.

In the VMRT we constructed, the participants' task is to decide as fast as possible whether two presented stimuli are identical or mirror images of each other while keeping errors to a minimum. Stimuli are 12 Shepard and Metzler (1971) like block figures, which are composed of 10 blocks with 4 branches (see Figure 1). The stimuli are monoscopic yet three-dimensional perspective renderings. The stimuli are bright green coloured and presented in front of a black background. An upright standing standard figure is presented with one comparison figure, which is rotated in the picture plane and either identical ("same" trials) or, in half of the trials, mirror reversed ("different" trials). The standard figure is presented on the left side and the comparison figure on the right. The angular disparity between the two figures is either $22.5^{\circ}, 67.5^{\circ}, 112.5^{\circ}$, or $157.5^{\circ}$ clockwise as well as counterclockwise (i.e., $202.5^{\circ}, 247.5^{\circ}, 292.5^{\circ}$, or $337.5^{\circ}$ ). Each comparison figure is presented twice (once as "same" and once as "different" figure) in each of the eight angular disparities which results in a total of 192 trials. Each trial starts with a $500 \mathrm{~ms}$ presentation of a grey $5 \mathrm{~mm}$ fixation square.

The VMRT was presented on a 17-inch monitor using the software 3D GameStudio A6. The software allowed for measuring reaction times in milliseconds. The input device was a two-button mouse.

Procedure. The experimental sessions lasted for approximately 35 minutes and took place in a laboratory of the Heinrich-Heine-University, Duesseldorf. Participants were tested in pairs and were paid for their participation. They were first given the MRT. After a 5 minute break they were tested with the VMRT. The participants were familiarised with the stimuli and their task in 24 practice trials. In this familiarisation phase, they received a "correct"|"false" feedback for each trial. None of the block figures used here was presented in the following experimental phase. The 192 trials of the experimental phase were divided into four blocks; blocks were separated by a break. Each trial started with the presentation of the fixation square followed by the two block stimuli prompting participants to answer by pressing either the left mouse button ("same") or the right button ("different").

Dependent variables and statistical analysis. The overall score on the MRT was computed for each participant. In the MRT, one point is given if both choices on one item are correct. Thus the maximum score is 24 . 


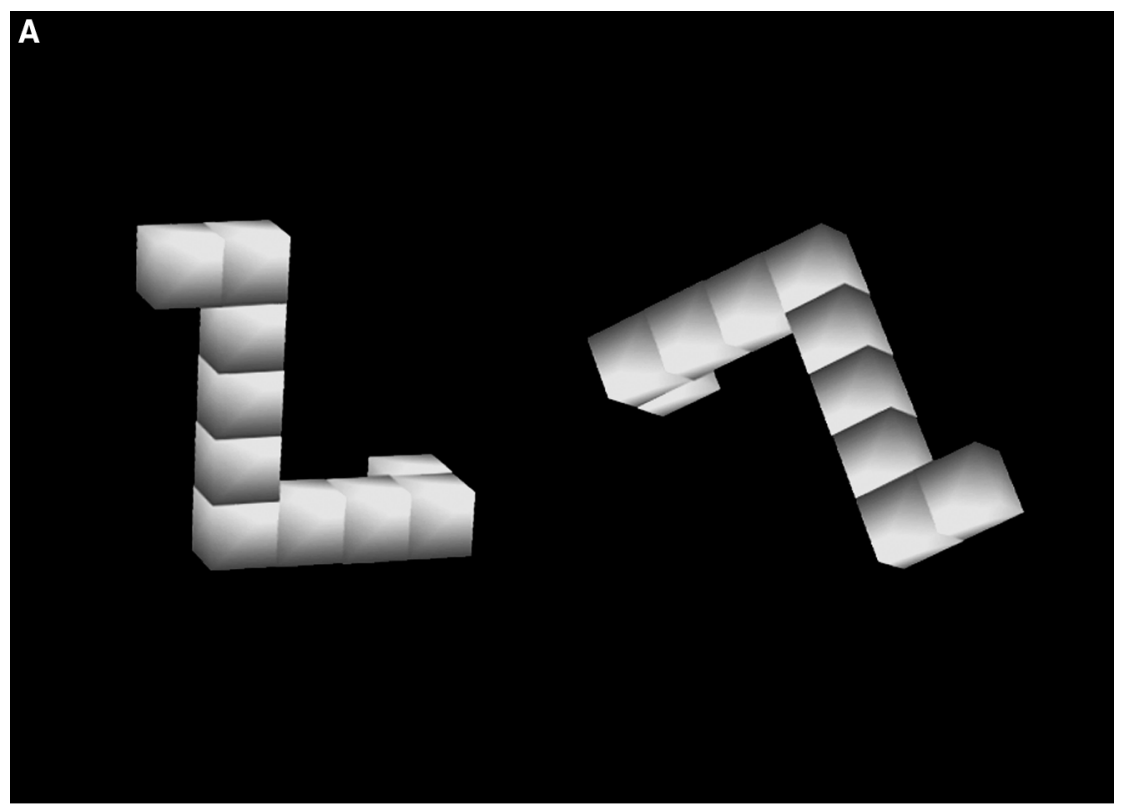

B
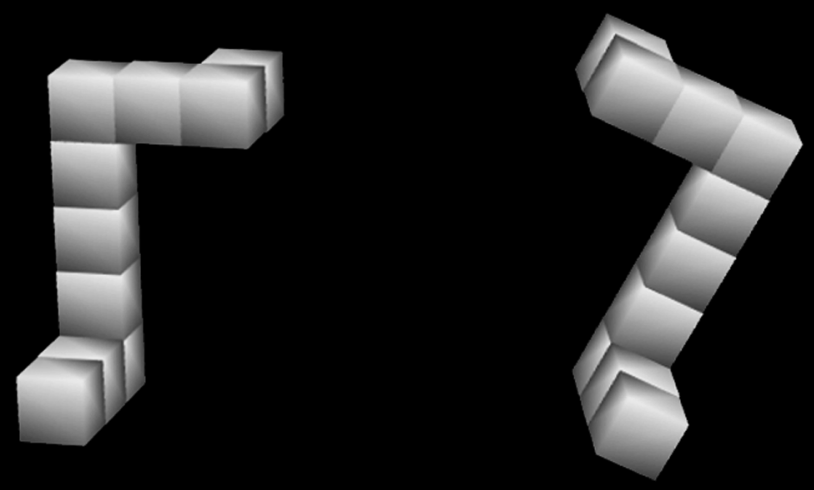

Figure 1. Example of block figures used in the VMRT. The standard figure is presented on the left side, the comparison figure on the right. (top) A "same" trial with an angular disparity of $157.5^{\circ}$. (bottom) A "different" trial with an angular disparity of $22.5^{\circ}$. 
There were two dependent variables in the VRMT: reaction time (RT) and errors. For each participant, the average RT in milliseconds was computed only for correct answers in "same" trials (see Shepard \& Metzler, 1971). In doing so, the RTs were clustered in four angular disparities: $22.5^{\circ}\left(22.5^{\circ}\right.$ and $\left.337.5^{\circ}\right), 67.5^{\circ}\left(67.5^{\circ}\right.$ and $\left.292.5^{\circ}\right), 112.5^{\circ}\left(112.5^{\circ}\right.$ and $\left.247.5^{\circ}\right)$, and $157.5^{\circ}$ $\left(157.5^{\circ}\right.$ and $\left.202.5^{\circ}\right)$. Within each cluster, and separately for each participant, RTs shorter than $500 \mathrm{~ms}$ and longer than two standard deviations above the mean were discarded. The number of errors (for both "same" and "different" trials) was computed overall as well as separately for each of the four clusters.

Angular disparity was defined as a within-subject factor. ${ }^{1}$ Furthermore, regression lines (least squares lines) between angular disparity and RTs were computed separately for each participant. The fit of the regression lines $\left(R^{2}\right)$, the slopes, and the intercepts were averaged across participants.

Bivariate correlations of the MRT score with mean RT in the VMRT, the number of errors in the VMRT, as well as the slope and the axis intercept of the regression lines in the VMRT were computed.

\section{Results and discussion}

Mean overall score of the MRT averaged across participants was $M=12.51$ $(S D=4.9)$.

In the VRMT, angular disparity had a significant effect on RT, $F(3$, $303)=284.5, p<.001$. Repeated contrasts revealed significant differences between all angular disparities with longer RTs for higher angular disparities (see Figure 2).

The averaged $R^{2}$ of the regression lines was .91. The slope averaged across participants was $11.91 \mathrm{~ms} /$ degree (which corresponds to a mental rotation speed of 83.96 degree/s); the axis intercept was $1519.66 \mathrm{~ms}$.

The overall error rate in the VRMT was $11.84 \%$. The angular disparity had a significant influence on the mean number of errors, $F(3,303)=94.6$, $p<.001$. Repeated contrasts revealed significant differences between all angular disparities with increasing number of errors for higher angular disparities (see Figure 2).

The MRT score correlated significantly with the RT of the VMRT, $r=$ $-.43, p<.001$, and with the mean number of errors of the VMRT, $r=-.36$, $p<.001$. The higher the participants scored on the MRT the faster they answered and the fewer errors they made in the VMRT. Concerning the regression lines in the VRMT, the correlation between the MRT score and the slope marginally failed to reach significance, $r=-.19, p=.051$, while the

\footnotetext{
${ }^{1}$ Significance levels of all ANOVA results of Experiment 1 were corrected according to Greenhouse-Geisser to compensate for the nonsphericity of the data.
} 


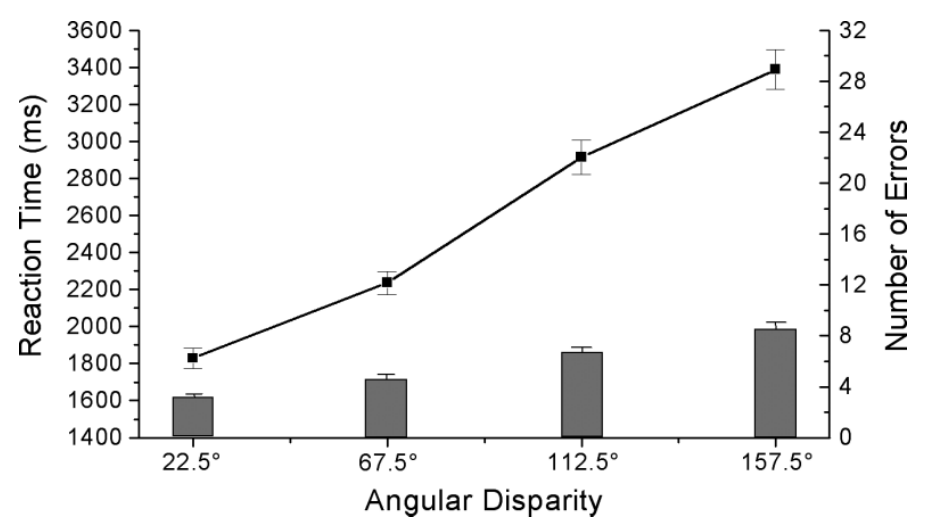

Figure 2. Mean reaction times (line graph) and mean number of errors (columns) depending on the angular disparity in the VMRT (Experiment 1). Error bars indicate standard errors.

correlation between the MRT score and the axis intercept was statistically significant, $r=-.39, p<.001$.

In summary, the results of the VMRT show a nearly perfect linearly increasing function between RT and the angular disparity. This is in line with the existing literature including the classic study of Shepard and Metzler (1971) and illustrates that participants use mental rotation to perform the task. Furthermore, participants' performance in the VMRT correlated significantly with the performance in the MRT. The higher participants scored in the MRT, the faster they were in the VMRT and the fewer errors they made. Analysing the reaction time in more detail by computing regression lines revealed that the MRT score correlated significantly with the axis intercept. While the slope is often associated with the mental rotation process itself, the intercept data seems to be based on other processes like stimulus encoding and comparison (e.g., Cooper \& Shepard, 1973; Kail, Pellegrino, \& Carter, 1980).

Overall, the VMRT could be validated successfully. The results of Experiment 1 clearly show that the VMRT is an appropriate computerbased test instrument for measuring mental rotation ability. Thus, the basis to investigate potential effects of manual training of mental rotation was established. This was done in Experiment 2.

\section{EXPERIMENT 2}

In Experiment $2,^{2}$ the effect of manual rotation training in a virtual reality on the mental rotation ability was studied. It was assumed that manual

\footnotetext{
${ }^{2}$ A short report of Experiment 2 is part of Wiedenbauer and Jansen-Osmann's (2005) conference paper.
} 
training would improve the mental rotation ability and it was examined whether this effect would be limited to previously learned stimuli. Furthermore, we assumed that participants with poor mental rotation ability would profit from manual training to a greater extent than good mental rotators (see Rizzo et al., 2001; Saccuzzo, Craig, Johnson, \& Larson, 1996).

\section{Method}

Participants. 67 subjects participated in Experiment 2 (33 were female), none of whom had participated in Experiment 1. Their age ranged from 19 to 45 years $(M=25.19)$. 61 participants were university students $(25$ of whom studied psychology) and the remaining 6 were employees. The vision of all subjects was corrected to 20/20. Three participants were dismissed due to an overall error rate in the VMRT of above $40 \%$. This resulted in 64 participants in total (half of whom were female).

Materials. The experiment was presented on a 17 -inch monitor using the software 3D GameStudio A6. The software measured reaction times in milliseconds. Input device was a two-button mouse and a Microsoft sidewinder precision joystick.

The VMRT of Experiment 1 was used as posttest. A parallel version was developed and used as pretest. This pretest contains six block figures that are similar but not identical to those of the VMRT. The stimuli are presented in the same angular disparities as in the VMRT. This results in a total of 96 trials (i.e., half the number of the VMRT posttest trials).

The participants' task in the virtual manual rotation training is to manually rotate the comparison block figure into the orientation of the standard figure. Similarly to the VMRT, the standard figure is presented on the left side and the comparison figure on the right side. Stimuli are 12 bright green coloured block figures consisting of 10 blocks. Half of the block figures are composed of four branches, the other half of five branches. To study object specific training effects, the block figures with four branches presented here are the same as in the VMRT posttest (which results in six "old" and six "new" block figures in the VMRT posttest). There are only "same" trials, that is standard and comparison figure are identical and only differ in the angular disparity in the picture plane. The angular disparities between the two presented objects are the same as in the VMRT. Each comparison object is presented in each of the eight angular disparities. Each trial is presented twice (but never consecutively), which results in a total of 192 trials.

In this experiment, the input device for rotating the comparison figure was a joystick. The joystick was embedded in a paperboard-box to allow for 


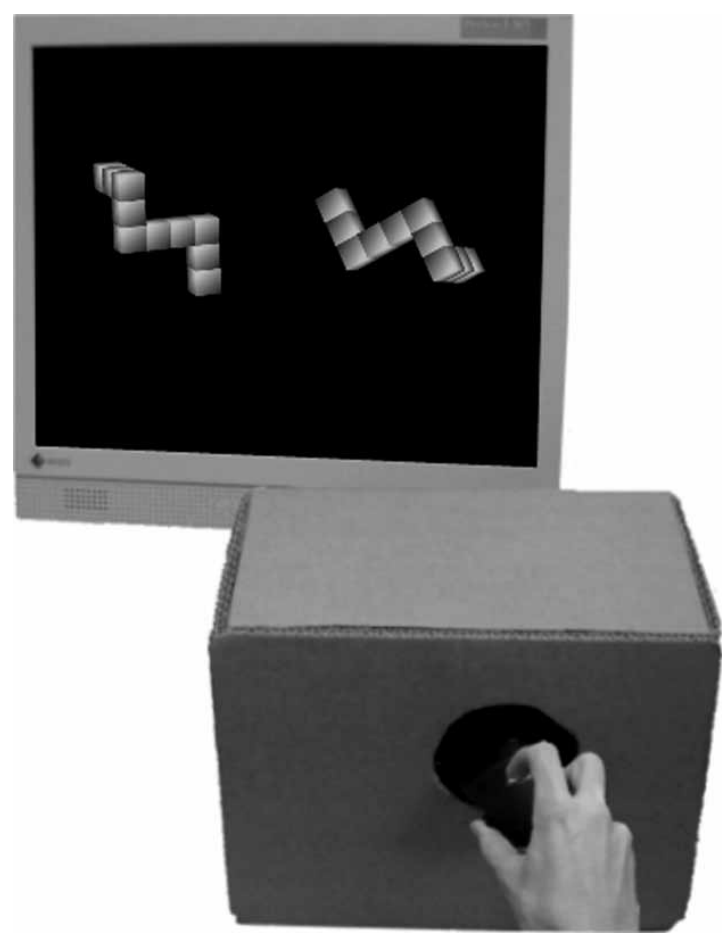

Figure 3. Stimuli and input device of the virtual manual rotation training.

grasping the joystick as one would do when rotating a real block figure in the picture plane (see Figure 3). When the joystick was turned right/left, the block figure in the virtual environment rotated in the corresponding direction.

The computer-quiz "Who wants to be a millionaire?" (German version) was used as a nonspatial filler task for participants in the nontraining group. In this quiz, a virtual quizmaster asks the participant questions, whereupon the player has to choose the correct answer out of four possibilities. The degree of difficulty increases from one question to the next. To ensure that participants took the task seriously and made an effort, they were told that the task was designed to test their general knowledge.

Procedure. The experimental sessions lasted for about 60 minutes and took place in a laboratory of the Heinrich-Heine-University, Duesseldorf. Participants were tested in pairs and paid for their participation. The participants of each pair were randomly assigned to the experimental and the control group. This resulted in a total of 32 participants per group (half of whom were females). The VMRT pretest started with a familiarisation 
phase (see Experiment 1) followed by the 96 experimental trials. Afterwards, the experimental group had to complete the virtual manual rotation training while the control group completed the computer-quiz for the same amount of time. The experimental group was shown how to handle the joystick and was instructed to rotate the comparison block figure into the spatial position of the standard figure and then to press the "fire button" of the joystick. If the spatial position of the standard and the comparison figure differed by more than 15 degrees to the right or to the left, the trial was registered as an error and the word "false" was presented in red letters. After the manual training and the computer-quiz, respectively, all participants had to perform the VMRT posttest.

Dependent variables and statistical analysis. Dependent variables were reaction time (RT) and errors. For each participant, the average RT in milliseconds was computed only for correct answers in "same" trials (see Shepard \& Metzler, 1971). RTs were clustered according to the four angular disparities: $22.5^{\circ}, 67.5^{\circ}, 112.5^{\circ}$, and $157.5^{\circ}$. Within each cluster, and separately for each participant, RTs shorter than $500 \mathrm{~ms}$ and longer than two standard deviations above the mean were defined as outliers and therefore discarded. The number of errors (for both "same" and "different" trials) was computed overall as well as separately for each of the four clusters.

The VMRT pretest data were analysed to assess baseline mental rotation ability. Angular disparity was defined as a within-subject factor ${ }^{3}$ for RT and errors. Furthermore, regression lines (least squares lines) between angular disparities and RTs were computed separately for each participant. The fit of the regression lines $\left(R^{2}\right)$, the slopes, and the intercepts were averaged across participants.

To assess training effects, differences in RT and errors were computed between pretest and posttest for each angular disparity (Note: To account for the different number of trials only half of the errors in the posttest were subtracted from the errors in the pretest). The difference scores of the experimental and control group were compared in order to study training effects. To achieve a more detailed analysis of the training effect, two additional independent variables were included in the analysis, namely the factor object and the factor mental rotation ability. To study object specificity, it was differentiated between trials with block figures that were trained in the manual rotation training ("old" objects) and trials with untrained block figures ("new" objects). To compare the training effect between participants with good and poor mental rotation abilities, both the

\footnotetext{
${ }^{3}$ Significance levels of all ANOVA results of Experiment 2 were corrected according to Greenhouse-Geisser to compensate for the nonsphericity of the data.
} 
experimental and the control group were divided by median split half according to their pre-test performance into good and poor mental rotators.

Angular disparity $\left(22.5^{\circ}, 67.5^{\circ}, 112.5^{\circ}\right.$, and $157.5^{\circ}$ ) and object (old vs. new) were defined as within-subject factors, whereas group (experimental vs. control group) and mental rotation ability (good vs. poor) were defined as between-subject factors.

Furthermore, regression lines (least square lines) between angular disparities and RTs for both pre- and posttest were computed for each participant. To analyse training effects, regression lines were averaged across participants depending on the experimental group. Slope and intercept in the pre- and posttest served as dependent variables and group was defined as between-subject factor. To analyse object specificity, regression lines of the posttest were averaged across participants depending on the group and object. The slope and intercept were compared between experimental and control group separately for "old" and "new" objects. To analyse training effects of good and poor mental rotators, regression lines of the posttest were averaged across participants depending on the group and mental rotation ability. The slope and intercept were compared between experimental and control group separately for poor and good mental rotators.

To asses manual rotation behaviour, rotation time in the manual rotation training was recorded and the correct trials were analysed separately for each of the four angular disparities. A trial was correct if the spatial position of the standard and the comparison figure differed by less than 15 degrees to the right or to the left. Within each angular disparity and separately for each participant, rotation times longer than two standard deviations above the mean were defined as outliers and therefore discarded. Angular disparity $\left(22.5^{\circ}, 67.5^{\circ}, 112.5^{\circ}\right.$, and $\left.157.5^{\circ}\right)$ was defined as a within-subject factor for rotation times. Furthermore, regression lines (least square lines) between angular disparities and rotation times were computed separately for each participant. The fit of the regression lines $\left(R^{2}\right)$, the slopes, and the intercepts were averaged across participants. Additionally, the mean overall error rate was recorded.

For the experimental group, the correlation between RT in the VMRT pretest and rotation time in the manual rotation training were computed. Also the slopes and intercepts between the VMRT pretest and the manual rotation training were correlated.

\section{Results and discussion}

Concerning the baseline mental rotation ability, the angular disparity had a significant effect on RT in the VMRT pretest, $F(3,189)=146.94, p<.001$. Repeated contrasts revealed a significant difference between all four angular 
disparities with higher RTs for higher angular disparities. The averaged $R^{2}$ of the computed regression lines was.86. The slope averaged across participants was $11.95 \mathrm{~ms} /$ degree ( 83.68 degree/s), the axis intercept was $1435.96 \mathrm{~ms}$.

The overall error rate was $12.4 \%$. Angular disparity had a significant effect on the number of errors, $F(3,189)=54.2, p<.001$. Repeated contrasts revealed significant differences between all four angular disparities with increasing number of errors for increasing angular disparities.

Concerning training effects, the RT difference scores differed significantly between the experimental and the control group, $F(1,60)=11.88, p<.05$. As can be seen in Figure 4, the RT difference score of the experimental group was much larger than that of the control group. The estimated size of the training effect for the mean difference score was $d=0.66$, which corresponds to a medium to large effect (according to Cohen, 1977). There was no effect of angular disparity on the RT difference score, $F(3,180)=2.98, p=.06$.

The RT difference score differed significantly between "old" and "new" objects, $F(1,60)=19.57, p<.001$. A significant interaction between object and group was found, $F(1,60)=14.01, p<.001$. Whereas the RT difference score for "new" objects did not differ between both groups, this was the case for "old" objects (see Table 1). Furthermore, there was a significant interaction between angular disparity and object, $F(3,180)=6.91, p<.05$ (repeated contrasts revealed a significant difference of the groups between angular disparities $112.5^{\circ}$ and $157.5^{\circ}$ ). Estimation of effect size revealed a large training effect for "old" objects $(d=0.93)$ and a medium effect for "new" objects $(d=0.44)$.

Regarding mental rotation ability, there were four post hoc groups: good mental rotators who received training (averaged pretest RT: $M=2006.85 \mathrm{~ms}$, $S E=75.65)$, poor mental rotators who received training $(M=2994.77 \mathrm{~ms}$,

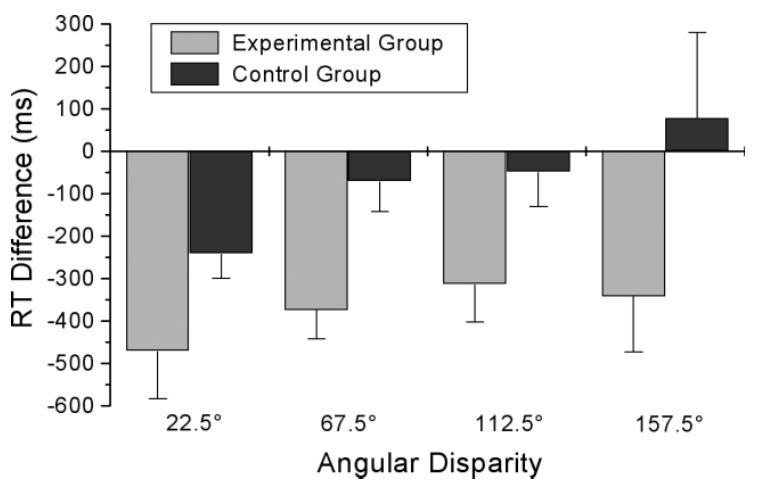

Figure 4. RT difference for the experimental and the control group depending on the angular disparity (Experiment 2). Negativity indicates faster RTs in the posttest. Errors bars indicate standard errors. 
TABLE 1

RT difference scores for the experimental and the control group depending on the angular disparity and separately for poor and good mental rotators as well as for

"old" and "new" objects (Experiment 2); negativity indicates faster RTs in the posttest (standard errors in parentheses)

\begin{tabular}{|c|c|c|c|c|c|c|c|c|}
\hline & \multicolumn{4}{|c|}{ Experimental group } & \multicolumn{4}{|c|}{ Control group } \\
\hline & $22.5^{\circ}$ & $67.5^{\circ}$ & $112.5^{\circ}$ & $157.5^{\circ}$ & $22.5^{\circ}$ & $67.5^{\circ}$ & $112.5^{\circ}$ & $157.5^{\circ}$ \\
\hline $\begin{array}{l}\text { Poor } \\
\text { rotators }\end{array}$ & $\begin{array}{r}-698.23 \\
(207.1)\end{array}$ & $\begin{array}{r}-536.92 \\
(109.8)\end{array}$ & $\begin{array}{r}-532.69 \\
(136.72)\end{array}$ & $\begin{array}{l}-567.29 \\
(223.41)\end{array}$ & $\begin{array}{r}-408.04 \\
(85.72)\end{array}$ & $\begin{array}{r}-142.83 \\
(129.96)\end{array}$ & $\begin{array}{r}-126.86 \\
(153.63)\end{array}$ & $\begin{array}{r}121.29 \\
(388.56)\end{array}$ \\
\hline $\begin{array}{l}\text { Good } \\
\text { rotators }\end{array}$ & $\begin{array}{r}-245.42 \\
(47.16)\end{array}$ & $\begin{array}{r}-213.92 \\
(54.67)\end{array}$ & $\begin{array}{r}-95.60 \\
(85.73)\end{array}$ & $\begin{array}{c}-118.18 \\
(120.58)\end{array}$ & $\begin{array}{l}-76.88 \\
(50.43)\end{array}$ & $\begin{array}{r}-0.60 \\
(55.55)\end{array}$ & $\begin{array}{r}27.29 \\
(52.49)\end{array}$ & $\begin{array}{r}35.45 \\
(125.0)\end{array}$ \\
\hline $\begin{array}{l}\text { Old } \\
\text { objects }\end{array}$ & $\begin{array}{r}-486.39 \\
(105.82)\end{array}$ & $\begin{array}{r}-421.13 \\
(72.20)\end{array}$ & $\begin{array}{r}-249.11 \\
(83.25)\end{array}$ & $\begin{array}{l}-279.96 \\
(131.18)\end{array}$ & $\begin{array}{r}-165.03 \\
(55.93)\end{array}$ & $\begin{array}{r}22.52 \\
(96.15)\end{array}$ & $\begin{array}{r}119.69 \\
(78.37)\end{array}$ & $\begin{array}{r}459.20 \\
(259.32)\end{array}$ \\
\hline $\begin{array}{l}\text { New } \\
\text { objects }\end{array}$ & $\begin{array}{r}-468.47 \\
(121.52)\end{array}$ & $\begin{array}{r}-345.50 \\
(74.89)\end{array}$ & $\begin{array}{r}-321.80 \\
(101.39)\end{array}$ & $\begin{array}{r}-397.11 \\
(132.02)\end{array}$ & $\begin{array}{r}-299.45 \\
(59.41)\end{array}$ & $\begin{array}{r}-143.41 \\
(70.70)\end{array}$ & $\begin{array}{r}-139.40 \\
(96.96)\end{array}$ & $\begin{array}{l}-136.15 \\
(168.77)\end{array}$ \\
\hline
\end{tabular}

$S E=168.36)$, good mental rotators who received no training $(M=1975.66$ $\mathrm{ms}, S E=71.61)$, and poor mental rotators who received no training $(M=$ $3118.89 \mathrm{~ms}, S E=180.61)$. Good and poor mental rotators differed significantly in their RT difference scores (see Table 1), $F(1,60)=7.17$, $p<.05$. There was no interaction between mental rotation ability and group, $F(1,60)=2.67, p=.108$. The estimated effect sizes revealed a large training effect (according to Cohen, 1977) for both good $(d=0.83)$ and poor $(d=$ $0.77)$ mental rotators. Furthermore, there was a significant interaction for mental rotation ability and object, $F(1,60)=14.01, p<.05$.

The regression lines in the pre- and posttest of the experimental group differed from those of the control group neither in intercept [pretest, $F(1,62)=$ $1.0, p=.33$; posttest, $F(1,62)=1.9, p=.18$ ] nor in slope [pretest, $F(1,62)=0.3$, $p=.6$; posttest, $F(1,62)=2.0, p=.16$ ] (see Table 2 for the posttest data).

TABLE 2

Axis intercepts and slopes for the experimental and the control group in the posttest (Experiment 2) in total, separately for poor and good mental rotators, and separately for "old" and "new" objects

\begin{tabular}{lcclcc}
\hline & \multicolumn{2}{c}{ Experimental group } & & \multicolumn{2}{c}{ Control group } \\
\cline { 2 - 3 } \cline { 5 - 6 } & Intercept $(m s)$ & Slope (ms/degree) & & Intercept (ms) & Slope (ms/degree) \\
\hline Total & 1024.67 & 12.22 & & 1138.8 & 14.86 \\
Old objects & 971.88 & 12.99 & & 1122.19 & 17.05 \\
New objects & 1059.64 & 11.75 & & 1128.69 & 13.77 \\
Poor mental rotators & 1092.72 & 14.65 & & 1221.11 & 19.55 \\
Good mental rotators & 956.63 & 9.80 & & 1056.48 & 10.17 \\
\hline
\end{tabular}


Concerning "old" objects, the regression lines in the posttest neither differed in intercept, $F(1,62)=2.3, p=.13$, nor in slope, $F(1,62)=3.0, p=$ .09 , between the two groups (see Table 2 ). The same pattern of results was found for new objects, $F(1,62)=0.6, p=.44$ for intercept, $F(1,62)=1.5, p=$ .23 for slope.

The regression lines of the poor and good mental rotators did not differ significantly between the experimental and control group (see Table 2). This was true for axis intercept, $F(1,30)=1.7, p=.2$, and slope, $F(1,30)=0.1$, $p=.78$, of good mental rotators as well as the for axis intercept, $F(1,30)=$ $0.78, p=.36$, and slope, $F(1,30)=2.7, p=.11$, of poor mental rotators.

The difference scores for errors depending on the four angular disparities and the group are shown in Table 3. Difference scores for errors did not differ between the two groups, $F(1,60)=1.34, p=.25$. Angular disparity had a significant influence on the difference scores for errors, $F(3,180)=2.97$, $p<.05$, whereas repeated contrasts did not reveal any significant differences. There were no other significant effects or interactions on error difference scores.

Looking at the manual rotation behaviour, angular disparity had a significant effect on rotation time in the manual training, $F(3,93)=838.4$, $p<.001$. Repeated contrasts revealed significant differences between all consecutive angular disparities. Rotation time increased linearly with increasing angular disparity between the two objects, the averaged $R^{2}$ for regression lines was .97 . The slope averaged across participants was $23.0 \mathrm{~ms} /$ degree (43.48 degree/s), the axis intercept was $1937.64 \mathrm{~ms}$. The overall error rate in the virtual manual rotation training was $1.24 \%$.

The correlation between RT for the mental rotation (VMRT pretest) and the rotation time of the manual rotation was statistically significant, $r=.37$, $p<.05$. The faster participants were in the VMRT pretest, the faster they were in the manual rotation. Furthermore, there was a significant correlation between the slope of the VMRT pretest and the slope of the manual rotation, $r=.44, p<.05$, as well as between the intercepts, $r=.36, p<.05$.

To summarise, in the pretest of the VMRT a nearly perfect linearly increasing function between RT and the angular disparity was found, which illustrates that participants use mental rotation to perform the task. The same

TABLE 3

Difference scores of mean errors (standard errors in parentheses) for the experimental and the control group depending on the angular disparity (Experiment 2); negativity indicates fewer errors in the posttest

\begin{tabular}{lrrrr}
\hline & $22.5^{\circ}$ & $67.5^{\circ}$ & $112.5^{\circ}$ & $157.5^{\circ}$ \\
\hline Experimental group & $-0.39(0.21)$ & $0.17(0.26)$ & $-0.58(0.40)$ & $-1.66(0.42)$ \\
Control group & $-0.38(0.26)$ & $-1.10(0.35)$ & $-1.53(0.49)$ & $-1.06(0.46)$ \\
\hline
\end{tabular}


function between rotation time and the angular disparity was found in the manual training as well. The comparison of the RT difference scores between experimental and control group revealed an effect of the manual training. Poor as well as good mental rotators profited from the manual training to a great extent. However, the RT difference scores differed only for "old" objects between the experimental and the control group, which suggests that the training effect is limited to objects learned in the training. Furthermore, a close relation between mental and manual rotation was found.

\section{GENERAL DISCUSSION}

The main purpose of these experiments was to study the effect of manual training in a virtual reality environment on mental rotation ability. The results of Experiment 1 showed that the virtual mental rotation test (VMRT) we constructed is a valid test instrument: A linear increase of the reaction time depending on the angular disparity between the two virtual objects illustrates the mental rotation process, and dependent variables - that is reaction time and errors - highly correlated with the score of the MRT (Vandenberg \& Kuse, 1978). The same linear relation between reaction time and angular disparity was found in a parallel version of the VMRT that served as pretest in Experiment 2. Furthermore, the number of errors increased for higher angular disparities, in both Experiments 1 and 2 (see, e.g., Cooper, 1975; Voyer, 1995; Wraga et al., 2003).

Experiment 2 revealed that the manual training we constructed does indeed improve the mental rotation ability measured by the VMRT. The difference scores of reaction times between pre- and posttest were larger for the experimental than for the control group. However, the manual training did not reduce the number of errors, which is in line with studies that did not find an effect of mental rotation training on error rates (see, e.g., Leone, Taine, \& Droulez, 1993). As the difference score of errors was not higher for the training group than for the control group we can exclude the possibility that the training effect on reaction time is due to a speed-accuracy tradeoff. A differential analysis of the training effect revealed that both poor as well as good mental rotators profited in about the same manner from the manual training. This is in contrast to a study of Saccuzzo et al. (1996) in which less proficient mental rotators (here women) improved to a greater degree than better ones (here men) by practising mental rotation. This large improvement was found only when practice effects were examined on a computerised mental rotation task and not on a paper and pencil rotation task.

To investigate the basis of the training effect, a more detailed analysis of reaction times was performed. The differentiation between trained and untrained objects revealed only a training effect for objects presented in the 
manual training. Thus the training effect of manual training seems to be object specific similar to the effect of mental rotation training (Heil et al., 1998; Tarr \& Pinker, 1989), which indicates that effects of manual training on mental rotation might also be memory based. It remains to be investigated, however, what is stored and later retrieved from memory: The finding that the linear relation between reaction time and angular disparity almost vanishes with extensive mental rotation training (e.g., Tarr \& Pinker, 1989) and the orientation specificity of the training effect (Heil et al., 1998) suggest that possibly the exact stimulus configurations presented are stored in memory. This would increase the probability of direct memory access and make the mental rotation process unnecessary to solve the task. In manual training, however, the presented stimuli are indeed rotated and therefore experienced in various orientations. This should result in the storage of an orientation-free object representation, which would make the process of mental rotation unnecessary to solve the task. In Experiment 2 an object specific training effect was observed, but a typical mental rotation function was still found and the comparison of regression lines for old objects between the training and control group did not reveal a statistical significant difference of the slope. Therefore, we can conclude that the training did not result in an orientation-free representation of trained objects, which is sufficient to replace the mental rotation process by direct memory access in the following mental rotation test. Thus, it cannot be inferred from the object specificity of the training effect that mental rotation is replaced by memory retrieval. As participants still have to mentally rotate to solve the task, it seems likely that all processes executed during a mental rotation task benefit from the memory representation and the resulting familiarity of trained objects. Cooper and Shepard (1973) assumed that reaction times in a mental rotation task are composed of the time needed for the following processing stages: stimulus encoding, mental rotation of the stimuli, comparison of the stimuli, and motor response. The stage of stimulus encoding is further subdivided into two processes: stimulus identification and the search for corresponding segments in the standard and the comparison figure in order to determine the orientation of the comparison figure. We suppose that the familiarity of the trained objects does simplify these processes. Regression lines were analysed to further investigate which of these processes are influenced by the manual training. A decline of the slope of the regression lines would indicate an acceleration of the mental rotation process, a decline in the intercept a speed up of other processes. As the training effect is reflected neither in the slope nor in the axis intercept, this does not allow for any conclusions.

Still, there is an alternative explanation for the object specificity of the training effect. As can be seen in Table 1, the difference scores of the reaction times for the control group differed between "old" and "new" objects, which 
is astonishing as participants were not given any training and consequently all objects were new. This implies that the objects used in the training were somehow more difficult than the untrained objects. This is confirmed by a post hoc analysis of Experiment 1: Reaction times for objects labelled as "old" in Experiment 2 were longer than for "new" objects, $t(101)=9.02, p<$ .001 . This difference in difficulty is coincidental as all objects were randomly assigned to the two groups. It is, however, not problematic for the analysis of the training effect per se as a training effect is defined as the difference between the difference-scores of the experimental and the control group. The lack of a training effect for "new" objects could, however, be based on the fact that these stimuli were less complex as Bethell-Fox and Shepard (1988) found that rotation time after mental rotation training was still dependent on angular disparity but not on stimulus complexity. For complex stimuli in particular, reaction time decreased after training. Bethell-Fox and Shepard suggested that complex stimuli could not be transformed in a holistic way. In the course of the training, however, participants learned to rotate complex stimuli holistically and thus complexity had no effect on reaction times in the posttest. In the study presented here, objects labelled as "old" seemed to be more complex than "new" objects. The manual training could induce a holistic strategy due to the visualisation and practice of the rotation process in which the block figures are rotated as a whole. The experimental group therefore might have profited from the training for "old" objects in particular, while the control group still rotated those stimuli in the posttest piece by piece. The lack of a training effect for "new" objects could be based on the fact that these less complex stimuli were transformed holistically even by the control group. Note that this alternative explanation is not contradictory to the explanations above. Firstly, switching from a piece-by-piece to a holistic strategy is just one possible way of improving the mental rotation process. Secondly, it is likely that the increased familiarity of trained objects - and therefore memory processes - facilitate the acquisition of a holistic strategy.

It seems that mental rotation is a complex multilayer process that has to be further investigated. One possibility would be to analyse the data of the manual rotation in more detail than was done in this experiment. We could, however, show a linear increase of rotation time with increasing angular disparity in the manual rotation task as is usually found in mental rotation. Furthermore, there was a significant correlation between mental and manual rotation: The faster participants were in mental rotation, the faster they were in the manual rotation task. The computation of regression lines showed that the slope as well as the axis intercept correlated significantly between mental and manual rotation. These similarities are in line with the results of Wohlschlaeger and Wohlschlaeger (1998) and show the close relation between mental rotation and motor processes. Therefore, by analysing 
manual rotation in more detail it could be possible to gain a better understanding of the processes during mental rotation and the interindividual differences between participants. It would for example be interesting to measure the time from the onset of the stimulus presentation to the beginning of the rotation (as an indicator of the stimulus encoding process), the time of the manual rotation itself, and the time from the end of the rotation to the motor response (indicating the comparison process). This could provide information about the same processes in mental rotation.

The present experiments showed the effectiveness of manual rotation training but do not provide any evidence that the training effect is due to the manual component. The visualisation of the rotation process is also likely to have an effect, especially with respect to memory processes. Possibly, a mental rotation like process could contribute to the effectiveness of the training: It seems likely that subjects rotate the stimuli-at least partlyprevious to the manual rotation in order to find out the shorter direction of rotation. It would be of great interest to investigate the critical components of the training and their relative contributions to the effectiveness in further studies. This could be done by a comparison of different training groups like a mental rotation, a visualisation, and a motor training group, which would additionally provide information about the differential effectiveness of specific training tasks.

Original manuscript received November 2005

Revised manuscript received March 2006

First published online 5 July 2006

\section{REFERENCES}

Barnes, J., Howard, R. J., Senior, C., Brammer, M., Bullmore, E. T., Simmons, A., et al. (2000). Cortical activity during rotational and linear transformations. Neuropsychologia, 38, 1148 1156.

Bethell-Fox, C. E., \& Shepard, R. N. (1988). Mental rotation: Effects of stimulus complexity and familiarity. Journal of Experimental Psychology: Human Perception and Performance, 14, 12 23.

Cohen, J. (1977). Statistical power analysis for the behavioral sciences. New York: Academic Press.

Cooper, L. A. (1975). Mental rotation of random two-dimensional shapes. Cognitive Psychology, 7, $20-43$.

Cooper, L. A., \& Shepard, R. N. (1973). Chronometric studies of the rotation of mental images. In W. G. Chase (Ed.), Visual information processing (pp. 75-176). Oxford, UK: Academic Press.

Heil, M., Roesler, F., Link, M., \& Bajric, J. (1998). What is improved if a mental rotation task is repeated - the efficiency of memory access, or the speed of a transformation routine? Psychological Research, 61, 99-106.

Hietanen, J. K., \& Perrett, D. I. (1996). Motion sensitive cells in the macaque superior temporal polysensory area: Response discrimination between self-generated and externally generated pattern motion. Behavioural Brain Research, 76, 155-167. 
Kail, R., \& Park, Y. S. (1990). Impact of practice on speed of mental rotation. Journal of Experimental Child Psychology, 49, 227-244.

Kail, R., Pellegrino, J., \& Carter, P. (1980). Developmental changes in mental rotation. Journal of Experimental Child Psychology, 29, 102-116.

Kaushall, P., \& Parsons, L. M. (1981). Optical information and practice in the discrimination of 3-D mirror-reflected objects. Perception, 10, 545-562.

Kosslyn, S. M., DiGirolamo, G. J., Thompson, W. L., \& Alpert, N. M. (1998). Mental rotation of objects versus hands: Neural mechanisms revealed by positron emission tomography. Psychophysiology, 35, 151-161.

Larson, P., Rizzo, A. A., Buckwalter, J. G., van Rooyen, A., Kratz, K., Neumann, U., et al. (1999). Gender issues in the use of virtual environments. CyberPsychology and Behavior, 2, 113-123.

Leone, G., Taine, M. C., \& Droulez, J. (1993). The influence of long-term practice on mental rotation of 3-D objects. Cognitive Brain Research, 1, 241-255.

Marmor, G. S. (1975). Development of kinetic images: When does the child first represent movement in mental images? Cognitive Psychology, 7, 548-559.

Parsons, T. D., Larson, P., Kratz, K., Thiebaux, M., Bluestein, B., Buckwalter, J. G., et al. (2004). Sex differences in mental rotation and spatial rotation in a virtual environment. Neuropsychologia, 42, 555-562.

Peters, M., Laeng, B., Latham, K., \& Jackson, M. (1995). A redrawn Vandenberg and Kuse Mental Rotations Test: Different versions and factors that affect performance. Brain and Cognition, 28, $39-58$.

Rizzo, A., Buckwalter, J. G., Bowerly, T., McGee, J. S., van Rooyen, A., van der Zaag, C., et al. (2001). Virtual environments for assessing and rehabilitating cognitive/functional performance: A review of projects at the USC Integrated Media Systems Center. Presence: Teleoperators and Virtual Environments, 10, 359-374.

Saccuzzo, D. P., Craig, A. S., Johnson, N. E., \& Larson, G. E. (1996). Gender differences in dynamic spatial abilities. Personality and Individual Differences, 21, 599-607.

Shepard, R. N., \& Metzler, J. (1971). Mental rotation of three-dimensional objects. Science, 171, $701-703$

Shepard, S., \& Metzler, D. (1988). Mental rotation: Effects of dimensionality of objects and type of task. Journal of Experimental Psychology: Human Perception and Performance, 14, 3-11.

Tarr, M. J., \& Pinker, S. (1989). Mental rotation and orientation-dependence in shape recognition. Cognitive Psychology, 21, 233-282.

Vandenberg, S. G., \& Kuse, A. R. (1978). Mental rotations, a group test of three-dimensional spatial visualization. Perceptual and Motor Skills, 47, 599-604.

Voyer, D. (1995). Effect of practice on laterality in a mental rotation task. Brain and Cognition, 29, $326-335$.

Voyer, D., Voyer, S., \& Bryden, M. P. (1995). Magnitude of sex differences in spatial abilities: A meta-analysis and consideration of critical variables. Psychological Bulletin, 117, 250-270.

Wexler, M., Kosslyn, S. M., \& Berthoz, A. (1998). Motor processes in mental rotation. Cognition, $68,77-94$.

Wiedenbauer, G., \& Jansen-Osmann, P. (2005). Manual training of mental rotation. In K. Opwis \& I.-K. Penner (Eds.), Proceedings of KogWis05 (pp. 207-212). Basel, Switzerland: Schwabe Verlag.

Wohlschlaeger, A., \& Wohlschlaeger, A. (1998). Mental and manual rotation. Journal of Experimental Psychology: Human Perception and Performance, 24, 397-412.

Wraga, M., Thompson, W. L., Alpert, N. M., \& Kosslyn, S. M. (2003). Implicit transfer of motor strategies in mental rotation. Brain and Cognition, 52, 135-143. 